\title{
Missed the take-home message
}

\author{
Lisa Merry ${ }^{1}$
}

Received: 23 May 2017 / Accepted: 29 May 2017 /Published online: 2 August 2017

(C) Springer-Verlag GmbH Austria 2017

\section{Dear Editor}

Thank you for the letter. The reader was quite diligent and they are correct. The sentence they quote from our discussion section "...using the cut-offs of $9 / 10$ and 12/13, the 1-week EPDS accurately classified the majority of women at 16 weeks postpartum with elevated E PDS scores" is not quite right. It would have been better to say "... using the cut-offs of 9/10, the 1-week EPDS accurately classified the majority of women ... with elevated EPDS scores." Without getting into a debate about semantics, we do feel it was reasonable to make this latter statement. Looking at the sensitivity analyses in column 1, in Table 1, the results for the whole sample, and sub-groups, for EPDS scores at 16 weeks, ranged from 47 to $67 \%$. Only two results were less than $50 \%$ and this is in the context of a 4-month period, which is a significant time difference to have a high predictive validity.

We also do not feel that we misled readers. Despite making the above statement regarding the 12/13 cut-off, a few sentences down in the same section we stated: "...when the cut-off score of 12/13 was used, the sensitivity of the measure was far less adequate with significantly more missed women at 16 weeks with depressive symptomatology." Moreover, the conclusion of our study, as stated in the abstract and at the end of the article, was "Our findings suggest that refugee, asylumseeking, non-refugee immigrant, and Canadian born women at risk of postpartum depression may be identified early in the postpartum period such that secondary preventive interventions may be implemented." We did not overstate our findings or draw definitive conclusions.

This article belongs to the Topical Collection: The EPDS: Thirty Years On

Lisa Merry

lmerry@uottawa.ca

1 School of Nursing, University of Ottawa, Ottawa, ON, Canada
We would also like to reiterate here the potential clinical utility of our findings for the migrant population. Migrant women are at greater risk (two times more likely than native-born women) for postpartum depression (FalahHassani et al. 2015). They also have significant barriers in accessing health and social services (Ganann et al. 2012; Merry et al. 2011; Sword et al. 2006). Our findings suggest that we may be able to identify a substantial number of migrant women early in the postpartum period, who may be at risk of sustained depression while they are still engaged with the healthcare system. These women then may be followed-up for further assessment and possibly treatment. This was the take-home message of the article.

\section{References}

Falah-Hassani K, Shiri R, Vigod S, Dennis C-L (2015) Prevalence of postpartum depression among immigrant women: a systematic review and meta-analysis. J Psychiatr Res 70:67-82

Ganann R, Sword W, Black M, Carpio B (2012) Influence of maternal birthplace on postpartum health and health services use. J Immigr Minor Health 14:223-229

Merry LA, Gagnon AJ, Kalim N, Bouris SS (2011) Refugee claimant women and barriers to health and social services post-birth. Canadian Journal of Public Health/Revue Canadienne de Santé Publique:286-290

Sword W, Watt S, Krueger P (2006) Postpartum health, service needs, and access to care experiences of immigrant and Canadian-born women. J Obstet Gynecol Neonatal Nurs 35:717-727 